\title{
ADORATSKIJ AN KAUTSKY
}

In den Jahren vor dem ersten Weltkrieg wurde der SPD und ihrem wichtigsten Theoretiker, Karl Kautsky, in den sozialistischen Parteien der ost- und südosteuropäischen Länder eine besonders grosse Anerkennung gezollt. ${ }^{1}$ „Für uns Russen war die deutsche Sozialdemokratie die Mutter, das lebendige Beispiel. Wir idealisierten sie aus der Entfernung. Die Namen von Bebel und Kautsky sprach man andächtig aus." So beschrieb Trotzki viele Jahre später ${ }^{2}$ seine Haltung gegenüber der grössten Partei in der 2. Internationale, die - allerdings in verschiedenen Graden der Abstufung der Anerkennung - fast allen russischen Marxisten gemeinsam war. Selbst Lenin, der weder (wie etwa Akselrod und Rjazanov) damals mit Kautsky befreundet gewesen ist, noch (wie Trotzki) ihn jemals als seinen ,Lehrer" bezeichnet hat, schätzte die theoretischen Arbeiten Kautskys hoch ein, wie er diesem im November I9I I noch brieflich bestätigte. Eine grundlegende Änderung in Lenins Urteil über Kautsky ist erst in den letzten Vorkriegsjahren, öffentlich gar erst im Winter I913/14 deutlich wahrzunehmen. ${ }^{3}$

$\mathrm{Zu}$ den russischen Sozialisten, die sich rat- und hilfesuchend an Kautsky wandten, gehörte auch V. V. Adoratskij, der nach 1918 eine führende Stellung unter den kommunistischen Gesellschaftswissenschaftlern einnahm. Seine Briefe an Kautsky ${ }^{4}$, die anschliessend zum ersten Male veröffentlicht werden, entsprechen der durchschnittlichen Haltung vieler russischer Marxisten gegenüber Kautsky vor dem Ersten Weltkrieg. - Vladimir Viktorovič Adoratskij (7. August 1878 - s. Juni 1945) stammte aus Kasan an der Wolga. ${ }^{5}$ Als is-16-

1 Vgl. E. Matthias, „Kautsky und der Kautskyanismus”, in: Marxismusstudien, 2. Folge, Tübingen 1957, S. 152; H. Brill, „Karl Kautsky”, in: Zeitschrift für Politik N.F. I (1954), S. 229, 236, 239; D. Geyer, „Die russ. Parteispaltung...”, in: IRSH III (1958), S. 195-219, $418-444$.

2 Leo Trotzki, Mein Leben. Versuch einer Autobiographie, Berlin I930, S. 203.

3 Vgl. z.B. W.I. Lenin, Sämtliche Werke, Bd. X, S. 284, 314 ff.; Bd. XII, S. 366 ; Bd. XVII, S. I3 Iff., I 47 f., 246; ders., Werke, Bd. 35, Berlin 1962, S. 108, 137 .

- Aus dem Kautsky-Achiv im IISG (D I, 274-276).

"Vgl.: P. Konjušaja, „Biografičeskij očerk”, in: V.V. Adoratskij, Izbrannye proizvedenija (Ausgew. Schriften), Moskau 196r, S. 598-61 1, sowie ebd., S. 59r ff.; Filosofskaja Enciklopedija, Bd. I (1960), S. 25; Adoratskij, „Za 18 let”, in: Proletarskaja Revoljucija, 1924, Nr. 3 (26), S. 92-I 16; N. Krupskaja, Erinnerungen an Lenin, 2. Aufl. Berlin 1960, S. 200 f. 
jähriger Schüler machte er Bekanntschaft mit den Werken Darwins, Häckels und Feuerbachs. 1897 liess er sich an der Universität seiner Heimatstadt immatrikulieren, wo er zunächst Mathematik, dann Rechtswissenschaft studierte. Während des Studiums lernte er das "Kommunistische Manifest" und andere Schriften von Marx und Engels kennen; 1900 schloss er sich der illegalen sozialdemokratischen Organisation Kasans an. Nach dem Abschluss seines Studiums im Jahre 1903 reiste er ins Ausland, wo er Anfang 1904 mit Lenin zusammentraf, der ihn für die Bolschewiki gewann. Im Mai I 904 kehrte Adoratskij nach Kasan zurück; dort nahm er nun - neben J. M. Sverdlov, dem späteren ersten Vorsitzenden des Allrussischen Zentralen Exekutivkomitees - eine führende Stellung in der örtlichen Parteiorganisation ein. Im gleichen Jahr erschien seine erste Schrift „Lehrplan der Hauptprobleme des Marxismus”. Zu Beginn des Jahres i 905 unternahm er abermals eine kurze Auslandsreise, auf der er Lenin besuchte. Im Dezember i905, nach dem Scheitern der ersten Revolution, wurde Adoratskij verhaftet und zunächst ins Gouvernement Astrachan verschickt, bis er schliesslich im Herbst 1906 die Erlaubnis erhielt, die zweijährige Verbannungszeit im Ausland zu verbringen. Während seines Aufenthalts in der Schweiz studierte er eifrig u.a. die Schriften von Marx, Engels und Mehring. Auch diesmal suchte er Lenin auf, der zu jener Zeit (1908) in Genf wohnte. Nach seiner Rückkehr nach Kasan im Jahre 1908 begann Adoratskij mit der Vorbereitung der im ersten Brief erwähnten Arbeit über den Staat, „vom materialistischen Gesichtspunkt aus betrachtet". I9I I reiste er über Paris und Berlin nach London (s. Brief 2). Im Winter I9II/I 2 nahm er in der Angelegenheit des sogen. „Russischen Fonds"1 im Auftrag Lenins Kontakt mit Kautsky auf. Nach eigenen fruchtlosen Gesprächen mit Kautsky und Bebel beauftragte Lenin Adoratskij, als Abonnent der „Vossischen Zeitung” einen Stuttgarter Advokaten für einen Prozess gegen Kautsky wegen der Schiedgerichtssache zu besorgen. ${ }^{2}$

Vom Frühjahr 191 2 bis zum Sommer 1914 war Adoratskij wieder in Russland. Auf einer erneuten Auslandsreise wurde er vom Kriegsausbruch in Deutschland überrascht; mit seiner Familie wurde er dort in den nächsten Jahren festgehalten (s. Brief 3). Erst im August i9I 8 konnte er nach Russland zurückkehren, wo ihm sofort wichtige Aufgaben auf wissenschaftlich-organisatorischem Gebiet übertragen

1 Vgl. L. Schapiro, The Communist Party of the Soviet Union, New York I960, S. 11 7-1 29. 2 Adoratskij, Proizvedenija, a.a.O., S. 591-593 (geringfügig anders in: Proletarskaja Revol., a.a.O., S. 100-102). - Man wandte sich an die Rechtsanwälte Dr. Kahn und Dr. Zürndorfer in Stuttgart. Zu einem Prozeß ist es nicht gekommen. 
wurden ${ }^{1}$ : Adoratskij wurde ins Volkskommissariat für Bildungswesen berufen und mit der Verwaltung des ehem. Hausarchivs der Zaren betraut sowie in November I9 8 zum Mitglied der - später so genannten - Kommunistischen Akademie ernannt. 1919 wurde er Dozent an der Universität Kasan, I920 (bis I925) Mitglied des Instituts für Parteigeschichte(Istpart) beim Zentralkomitee der RKP(B), stellvertretender Leiter der zentralen Archivverwaltung sowie Professor am Institut der Roten Professur, 1928 stellvertretender Direktor des LeninInstituts, I 93 I (bis 1938) Direktor des Marx-Engels-Lenin-Instituts², im gleichen Jahre Direktor des Philosophischen Instituts der Kommunistischen Akademie, 1936 (bis 1939) Direktor des Instituts für Philosophie der Akademie der Wissenschaften der UdSSR, als dessen wissenschaftlicher Mitarbeiter er 194 I-I 944 tätig war. In den Jahren I 93 8-I 94 I und 1944/45 war Adoratskij Hauptredakteur der wissenschaftlichen Ausgabe der Werke von Marx und Engels. Die Arbeit an einer von ihm geplanten grossen Marx-Biographie hat er nicht vollenden können.

$\mathrm{Da}$ Adoratskij stets ein „linientreuer" Kommunist gewesen ist, nimmt es nicht wunder, dass er, nachdem Lenin sich heftig gegen Kautsky gewandt hatte ${ }^{3}$, in ähnlicher Weise den früher als Lehrer Verehrten angriff. So warf Adoratskij Kautsky wie den anderen „Renegaten der 2. Internationale" (Otto Bauer, Hilferding, Vandervelde usw.) vor, sie hätten die Marx'sche Dialektik in Sophistik umgewandelt. ${ }^{4}$ I 932 beschuldigte er Kautsky, ,der die Eigenschaft der meisten Professoren hatte, über Dinge zu schreiben, ohne sie ganz zu verstehen", dass er von Demokratie "fasele" und nunmehr „das Vorbild eines verbissenen Apologeten der Bourgeoisie... ist, der sich vollständig vom dialektischen Materialismus, den kein Philister imstande ist, ganz zu erfassen, losgesagt hat". ${ }^{5}$ Diese Verurteilung durch

${ }^{1}$ Für Lenins Interesse an Adoratskij vgl.: Leninskij Sbornik Bd. 16, S. 97 f., Bd. 20, S. 349, Bd. 23, S. 315 f., Bd. 24, S. 291, Bd. 35, S. 34I f., Bd. 36, S. xor f., 313,324 f., 469 f. (kurze Charakteristik Adoratskijs); Lenin, Werke, Bd. 35, Berlin 1962, S. 420, 493.

${ }^{2}$ Nach der Absetzung und Verbannung des Direktors des Marx-Engels-Instituts, D. I. Rjazanov (s. [B.I. Nikolaevskij], Letter of an Old Bolshevik, New York 1937, S. 34 f., nach Mitteilungen Bucharins: Socialističeskij Vestnik, No. 12, 1959, S. 246) wurden die beiden wissenschaftlichen Zentralinstitute 193 I miteinander verschmolzen.

${ }^{3}$ In seinem Brief vom 20.9.1918 an V. V. Vorovskij schrieb Lenin über ,schändlichen Unsinn”, „kindisches Gestammel” und „plattesten Opportunismus” Kautskys (Werke, Bd. 35, S. 338). Vor allem ist hier an Lenins Schriften „Staat und Revolution" und „Die proletarische Revolution und der Renegat Kautsky" zu erinnern.

${ }^{4}$ Vgl. W. Adoratski, „Marxismus-Leninismus und Dialektik”, in: Unter dem Banner des Marxismus V (1931), S. 319; Adoratskij, Proizvedenija, a.a.O., S. 473 ff., 524 f.

${ }^{5}$ Adoratskij in: Marx-Engels, Briefe an Bebel, Liebknecht, Kautsky u.a., Tl. I, MoskauLeningrad x933, S. XII-XIV; MEGA, I. Abt. Bd. 5, S. XVI, Bd. 6, S. XIXf.; K. Marks, Izbrannye proizvedenija, Bd. II, o.O. 1934, S. 52 I (Anm.). - Instruktiv ist ein Vergleich der Einleitungen Rjazanovs und Adoratskijs zu den unter ihrer Leitung herausgegebenen MEGA-Bänden. 
Adoratskij ist ebenso typisch für die Haltung der Bolschewiki Kautsky gegenüber, nach der grossen Enttäuschung über das Verhalten der deutschen Sozialdemokratie am und nach dem 4. August I9I4, wie seine Briefe aus der Vorkriegszeit es sind für die Anerkennung aller Gruppen der russischen Sozialdemokratie gegenüber der grossen Bruderpartei und ihrem theoretischen Wortführer.

Grundsätzlich wurde der Originaltext mit seinen Stileigentümlichkeiten beibehalten; lediglich kleine grammatische Fehler (Artikel, Zeichensetzung u. dgl.) wurden stillschweigend berichtigt, grössere Aenderungen - mit Hilfe von [] oder Anmerkungen - besonders kenntlich gemacht.

Sehr Geehrter Lehrer, Ich schreibe Ihnen zum zweiten Male, welches vom ersten durch eine lange Periode von ungefähr 7 Jahre[n] geschieden ist. Es war im Jahre I904, als ich, noch ein grüner Jüngling, in Berlin kam. Ich bat Sie, mir einige Fingerzeige zu geben (über „Staat” und „Philosophie”). Sie waren damals so liebenswürdig, dass [Sie] mir antworteten. Dieser Umstand erlaubt zu hoffen, dass Sie auch jetzt mir einige Zeile[n] schreiben werden.

Es ist klar für mich, dass ausser Sozialismus gibt es keinen Ausgang aus der schweren Lage, in welcher die moderne Gesellschaft jetzt bleibt. Ich weiss sehr gut, das[s] eine einzige wissenschaftliche Waffe zum Erschliessen ${ }^{1}$ der Rätsel der gesellschaftlichen Fragen ist [die] Marx'sche dialectische Methode und seine materialistische Theorie der Gesellschaft. Aber es ist sehr [be]klagenswert, dass es kein[e] sozialistische Universität gibt, dass keine sozialistische[n] Seminare existieren, wo jeder seine mangelhafte[n] Kenntnisse des Sozialismus verbessern könnte.

Gewiss, es ist das Leben selbst, das als ein[e] Universität dienen muss. Und die Arbeit in der Partei ist auch sehr wichtig. Damit aber jemand die Lehren des Lebens sich nützlich machen könne, - es notwendig ist, schon eine gewisse Selbständigkeit (im Bereich der Theorie) zu haben im Gewinnen und Verdauen des rohen Material[s], der Facten. Und sehr oft kehrt das Leben zu dem Studierenden jene Seite, welche am wenigsten geeignet ist einige Litteraturangaben zu liefern oder Bücher, oder leitende Fingerzeige zu geben.

In unserem Falle überwiegend kehrte das Leben nicht die beste von

${ }^{1}$ Im Original: Entschließen. 
seinen Seiten zu uns. Es waren (diese Lebens-phänomene) provinzielle Lehrer, spiessbürgerliche und bureaucratische Professoren, viele von ihnen vom echt-russischen Muster, Pfaffen und als die höchste Academie Gendarmen und Polizei. Und dazu elendsten Lebensverhältnisse der russischen provinziellen Stadt des östlichen Russlands. Und trotzdem wurden wir Sozial-Demokraten. Das Leben nicht so stiefmütterlich war, um uns nur eine von seinen Seiten zu zeigen und wenn ,nur von Weitem" wehten zu uns andere Lüfte.

Als ich schon Student war in der Universität zu Kasan (so nennt sich meine Mutter-Stadt), las ich [das] Manifest von K. Marx. Das war mir ein Zeugniss von der besseren Welt, als jener in welcher ich selbst lebte. Gewiss konnte ich nicht das Manifest in seiner vollen ${ }^{1}$ Bedeutung verstehen; ich fühlte aber die Grossartigkeit des Werkes. Ich hatte keine Kenntnisse in der Geschichte und überhaupt sehr dürftige Kenntnisse auf allen Gebieten des Wissens, welche uns in Gymnasium und Universität dargeboten waren.

Wenn Marx die klägliche[n] Zustände des deutschen Unterrichtes [zu] verspotten und bedauern pflegte, wie uns Liebknecht in seinen „Erinnerungen”2 erzählt, - so glaube ich, dass unsere russische[n] noch tausendfach schlechter waren. Vor meiner Bekanntschaft mit [dem] Manifest und bald nachdem habe ich einige von Ihren Schriften gelesen und zwar diese in russischer Übersetzung erschienen haben bis $1902 \mathrm{Jahr}^{3}$ Als ich schon im letzten Semester war im Beginne des Jahres I 903, las ich Ihr „Erfurter Programm”4 und nur im Sommer I 903 die "Agrarfrage"4 in [einer] verstümmelten ersten russischen Ausgabe. Ich schreibe dies um Ihnen zu zeigen den Gang der Entwickelung des russischen Intellectuellen, der keine besonder[en] Talente hatte, aber wissens[be]dürftig war und ideelle Interessen hatte.

Da kam ich zum Ende meiner Universitäts Studien und war bereit ins Leben zu treten. Ich fühlte mich äusserst unwohl, da ich ohne jede Kenntniss war, die officielle Weisheit leugnend und die neue revolutionäre noch nur vorausahnend, aber nicht genau wissend. In diesem Zustande entschied ich, mich dem Studieren der russischen Rechtsgeschichte zu widmen, weil meine materielle[n] Existenz-Bedingungen ziemlich gut waren und mich nicht sofort zwangen mein Brot mit eigener Arbeit zu gewinnen. Dazu kam auch der Umstand, dass

1 Im Original: in voller seinen Bedeutung.

${ }^{2}$ W. Liebknecht, Karl Marx zum Gedächtnis. Ein Lebensabriß und Erinnerungen. Nürnberg I 896, S. 42.

${ }^{3}$ Vgl. W. Blumenberg, Karl Kautskys literarisches Werk, 's-Gravenhage i960.

- Die ersten russischen Úbersetzungen von Kautskys Büchern Das Erfurter Programm in seinem gtundsätzlichen Teil erläutert, Stuttgart I 892, und Die Agrarfrage, Stuttgart 1894, sind 1894 bzw. I 899 erschienen. 
einige jüngere Professoren und Privat-dozenten mir Rat gaben mich der wissenschaftlichen Arbeit zu widmen.

Die erste Frage welche sich vor mir stellte war die Frage: was der Staat ist? Nachdem ich [mich] vergebens bemühte, diese Frage selbständig zu lösen (Autorität der Ratgeber aus unserer Universität war für mich gewiss gleich null) kam ich zur Entscheidung, nach [dem] Ausland zu fahren und dort, besonders in Deutschland, weitere Kenntnisse von Marx' Lehren zu erwerben.

In Berlin kam ich im Januar 1904 [an]. Damals bat ich brieflich, dass Sie mir einige Fingerzeige gaben über Litteratur der Philosophie und was ist der Staat vom Standpunkte der Marx'schen Theorie. Sie antworteten mir und Thr Brief war mir sehr schätzbar, weil er war die erste Stimme vom lebendigen Menschen, dem ich glauben kann. Sie schrieben, dass eine persönliche Bekanntschaft mit mir für Sie nicht unerwünscht wäre, aber Sie fürchteten, dass Spitzel-Kursus der Preussischen Regierung mir schädliche Konsequenzen bereiten werden könne. Ich selbst suchte keine persönliche Bekanntschaft, weil ich damals keineswegs in guten Verhältnissen zur deutschen Grammatik und zur deutschen Sprache stand. Seitdem haben diese Verhältnisse [sich] beträchtlich verbessert, [sind] aber noch nicht zur vollen Eintracht gebracht. Bis jetzt kann ich nicht sprechen „fluently" und meine Rede mit grammatischen Fehler[n] wimmelt, wofür das beste Zeugniss dieser Brief ist. Nach zweimonatlichem Aufenthalt in Berlin, kam ich in die Schweiz und studierte die russische Sozial-demokratische Litteratur. Da bin ich ein Sozial-demokrat geworden und zwar der Richtung der Mehrheit (Bolschewiki). Die Umstände forderten, dass ich im Frühjahr heimkehrte. Im Sommer des Jahres 1904 begannen wir in unserer Mutterstadt Soz.-demokr. Agitation und Propaganda einzurichten (Ich und meine Freunde welche auch Studenten waren.) Das war halb Spiel - halb Ernst. Spiel - weil unsere gesellschaftliche[n] Verhältnisse am wenigsten dazu geeignet waren, [die] sozial-demokratische Bewegung zu begünstigen. Wir bewegten [uns] überwiegend in bürgerlichen Schichten, und das Material für unsere Einwirkungen war die studierende Jugend, welche auch von bürgerlichen und kleinbürgerlichen Schichten stammte. Erst später gewannen wir Bekanntschaften mit Handarbeitern. Aber im Gebiete der Grossindustrie existieren in Kasan nur zwei grössere Anstalten, je 2-4000 Arbeite[r] jede. ${ }^{1}$ Und diese waren überhaupt ausser unserem Wirkungskreise. Es dauerte nicht lange. Im Dezember r 905 war ich verhaftet und in[s] Gouvernement Astrachan verbannt. Im Herbste 1906 bekam ich

${ }^{1}$ I 899 gab es in Kasan eine Stearin- und Seifenfabrik mit 2200 und eine Gerberei und Textilfabrik met 2420 Arbeitern (C. Lehmann u. Parvus, Das hungernde RuBland, Stuttgart $1900,5.64$ ). 
Erlaubniss von [der] russischen Regierung, Russland zu verlassen und die Zeit meiner Verbannung (2 Jahre) im Auslande zu verweilen.

$\mathrm{Da}$ ich keineswegs ein vollständiger und bis zum letzten Punkte bewusster Sozial-demokrat war - verwirrte [sich] alles in meinem Kopfe. Ich hatte sehr mangelhafte Kentnisse im Gebiete der Geschichte und Sozialwissenschaften, und als ich in die Schweiz am Ende 1906 Jahr kam, fühlte ich mich äusserst unwohl. Da war eine Reaction ich begann $a^{1}$ allem [zu] zweifeln. Nur eins stand für mich felsenfest der historische Materialismus. Aber ich war noch nicht in voller Klarheit darüber. Ich glaubte, dass ich den historischen Materialismus verstanden habe, aber jeder Versuch der selbständigen wissenschaftlichen Arbeit missglückte. Es fehlten die philosophische[n] Kenntnisse und ich fühlte, dass ich keineswegs [die] Marx'sche Methode handhaben könne.

Ich studierte damals (im Jahre 1907) die deutsche und englische Sprache und staatswissenschaftliche Litteratur, aber immer blieb in Umwegen und Verwirrungen, da ich immer auf eigene Faust arbeiten wollte. Das Studieren der bürgerlichen Gelehrten zeigte mir, was für elendste Zustände die bürgerliche Wissenschaft durchlebt.

Marx und seine Schüler standen immer turmhoch über alle[n] diese[n] Volkswirtschaftler[n], Politiker[n], Juristen u.s.w. [Die] Bürgerliche Soziologie schien mir an der Grenze des Scharlatanentum[s] und [der] Plattheit zu stehen. Als ich diese Arbeiten las, tauchte immer eine Frage vor mir auf: ${ }^{2}$ Dürfen wir überhaupt wissen? Und [es] schrien aus allen Ecken [die] verschiedensten Stimmen der bürgerlichen Theoretiker: Nein wir dürfen nicht.

Meine Unzufriedenheit dauerte, bis ich alle diese wissenschaftliche Litteratur zum Teufel schickte und von neuem Marx und Engels zu studieren begann. Dazu beschäftigte ich [mich] mit der Geschichte und mit der Geschichte der Philosophie. Erst nachdem habe ich mich sicherer gefühlt. Ich habe verstanden, dass Marx vor allem ein Philosoph war. Sehr viel gab mir Mehring's „Geschichte der deutsch. Sozialdemokratie" und „Aus sein[em] litter. Nachlass."3

Jetzt unternahm ich eine Arbeit über die Methode der Erforschung des Staates. ${ }^{4}$ Ich habe schon ein[en] Entwurf gemacht und theils meine Arbeit geschrieben, aber ich möchte noch sie durcharbeiten und die Litteraturübersicht, die im I. Kapitel ist, vervollständigen.

1 Im Original: im.

2 Im Original: eine Frage auftauchte immer for mich.

${ }^{3}$ F. Mehring, Geschichte der deutschen Sozialdemokratie, Stuttgart 1897/98, 2. Aufl. ebd. I903/04; Marx-Engels-Lassalle, Aus dem literarischen NachlaB, Hrsg. v. F. Mehring, Stuttgart 1902.

4 1923 veröffentlichte Adoratskij die Arbeit unter dem Titel: O Gosudarstve. (K voprosu o metode issledovanija.) 
Der Inhalt ist folgender:

I. Kritische Uebersicht der Litteratur.

Die Schriften der Fachgelehrten.

[Allgemeine Staatslehre, Political science, science politique, geschichtliche Litteratur welche politische Verhältnisse studiert, Ethnologie]

Ich gruppiere diese Litteratur in 3 Theile.

a. Juristen - wissenschaftlicher Wert gleich Null. Rehm, Jellinek, Duguit, Esmein, $\mathbf{1}$ etc.

$b$. Bürgerliche Soziologen. Besser, weil wissenschaftlicher, aber ungenügend. Gumplowicz, Ratzenhofer. ${ }^{2}$

c. Forscher der Geschichte, der Vorgesch. und Ethnologen. Das ist das beste was Gelehrte, auf bürgerlichem Standpunkte stehend, leisten können. Sammeln und systematisieren des Materials. Aber Sie ungefähr alle leiden immer vom wissenschaftlichen Nihilismus und fühlen eine panische Furcht vor der Theorie, ${ }^{3}$ wenn Sie nicht dumm [sind] und keine Revolutionäre sein woilen.

II. Eigner Standpunkt.

Politische Verhältnisse sind reale Menschenverhältnisse, welchen gewisse Vorstellungen des menschlichen Kopfes entsprechen. Unterlage der politischen Verhältnisse ist [die] Möglichkeit (und Notwendigkeit) der Exploitation.

Politische Vorstellungen sind gleich rechtlichen, religiösen und so weiter Vorstellungen nur rein ideologische aber keineswegs theoretische. Darum muss man klar scheiden:- Verhältnisse und Vorstellungen über die ersten. Verhältnisse welche überwiegend sind - oekonomische Verhältnisse. [Die] Vorstellungen aber muss man kritisch durchsehen und ihre kritische Entwicklungsgeschichte geben. Und never vergessen, dass diese Vorstellungen nur eine verkehrte Durchspiegelung der realen Zustände bieten. Der Erforscher des Staates muss immer reale Zustände im Auge halten und darum vor allem ein Historiker sein.

Ich glaube, dass dieser Standpunkt ist ein richtiger und so dachte kein anderer als Marx selbst.

III. Weiter versuchte ich mit Marx' eigenen Worten sein[e] Ansicht

1 Hermann Rehm (1862-1917), deutscher Staatsrechtslehrer, Prof. in Marburg (1891), Gießen u. Erlangen (1893), Straßburg (I903). - Georg Jellinek (I85 I-I91 I), deutscher Staatsrechtslehrer, Prof. in Basel (1883) und Heidelberg (1891). - Léon Duguit (1859I 928), französischer Staatsrechtslehrer, Prof, in Caen (1883) und Bordeaux. - Adhémar Esmein (1848-1913), franz. Staatsrechtslehrer, Prof. in Paris (1887).

${ }^{2}$ Ludwig Gumplowicz (1838-1909), poln.-österr. Soziologe, poln. Jungdemokrat, seit I 875/82 Prof. in Graz. - Gustav Ratzenhofer (I842-1904), östert. General, Philosoph und Soziologe.

Im Original: und einen panischen Furcht von Theorie fühlen. 
auf den Staat zu characterisieren. Dafür benützte ich entsprechende Stellen aus [dem] Kapital, I. und III. Bd., aus Anti-Dühring und Entstehung der Familie. ${ }^{1}$

IV. Nachdem gebe ich geschichtliche Beispiele, welche als Illustrationen und auch als Beweise dienen können. Der wichtigste ist eine unüberwindliche Tendenz, dass Herrschaftsgebiet[e] mit Wirtschaftsgebiet[en] zusammen[zu]fallen pflegen. Der feudale Partikularismus $[-]$ die bürgerliche einigende Tendenz. Zwei letzte Kapitel möchte ich der Erörterung über Theorie und Methode und über Dialektik widmen. Unter Theorie und Methode versuche ich eine richtige Ansicht auf [die] wichtige Rolle [zu geben,] welche [die] Theorie in der methodischen Forschung spielt. Unter Dialektik ich möchte für mich selbst eine klare Vorstellung über diesen Gegenstand machen. Vielleicht können Sie mir einige Fingerzeige darüber geben.

Ich weiss sehr gut, dass jede von diesen Fragen, über welche ich geschrieben habe, eine selbständige Dissertation fordert. Ich weiss auch sehr gut, dass meine Kräfte sehr ungenügend sind. Aber [das] sehr niedere Niveau der bürgerlichen Arbeiten über diese Gegenstände erlauben mir zu hoffen, dass ich nicht die schlechteste[n] Resultate von meinen Arbeiten erwarten muss.

Aber jetzt fühle ich, dass es die Zeit ist meinen enorm langen Brief zu schliessen. Es entsteht eine Frage ,What do you want?” oder „Wozu der Lärm?”

Es steckt im Menschen ein unausrottbares Bedürfniss, [mit] seinesgleichen ${ }^{2}$ in Wechselbeziehungen eintreten zu wollen, und gewöhnlich überall erwünscht wird, dass doch einige Menschen-Brüder existieren, welche dieselbe Gesinnung haben und von gleichen Interessen begeistern. Im meinem Leben, welches schon 32 Jahren dauerte, bin $^{3}$ ich sehr selten mit solchen Leuten zusammengekommen, ${ }^{4}$ welche mit mir gleich gesinnt waren ${ }^{5}$ und dazu höhere wissenschaftliche Bildung hatten.

Ich fühle mich ,ashamed”, dass ich [Sie] vielleicht eine[r] ${ }_{4}^{\frac{1}{4}}$ Stunde Ihrer Zeit beraubt habe.

Aber wenn Sie noch eine $\frac{1}{4}$ Stunde freier Zeit haben, so könnte ich zu Ihnen kommen, wenn ich nicht fürchtete, dass meine mangelhafte[n] Kenntnisse der deutschen Sprache ein beträchtliche[s] Hinderniss beıeiten werden, ein Gespräch zu führen. Ich will hier in Berlin 3 oder

\footnotetext{
1 Von Friedrich Engels.

2 Im Original: seinem gleichen.

Im Original: habe.

- Im Original: zusammengetreten.

- Im Original : haben,
} 
4 Tage bleiben und gehe dann nach London, um meine englische Sprache zu verbessern und englische geschichtliche und ethnologische Litteratur zu studieren.

Wenn Sie aber keine Zeit haben und überhaupt es für Sie unmöglich ist eine neue Bekanntschaft zu machen, so vielleicht Sie gestatten mir von England zu schreiben und Zeit finden mir einige Fragen zu beantworten.

Die erste Frage ist Dialektik. Was ist darüber in [der] Litteratur wenn nicht solche Werke [zu] rechnen wie Engels Anti-Dühring, Engels Ludwig Feuerbach und Hegels Phänomenologie des Geistes und Logik.

Die zweite Frage [ist,] einen Rat zu geben, wie die Geschichte [zu] studieren [ist]. Es wäre sehr wünschenswert, solche Kompendien zu haben wie Mehring's Deutsche Geschichte Ein Leitfaden... ${ }^{1}$

Die dritte Frage [betrifft] Ethnologie und Vorgeschichte.

Ich wohne jetzt hier in Berlin vom 5. bis 8. Januar im Hotel Westfälischer Hof, Neustädtische Kirchstrasse I 3. Berlin N.W.

Zum Schlusse bitte ich um Verzeihung für mein[en] barbarischen Styl und [meine] Fehler.

Mit Sozial-demokratischem Gruss

s/I I9г. Berlin

W. Adoratsky

53 Bernard Str. Russel Sq. London W.C. [Ende Januar I9I I]

Sehr Geehrter Genosse,

Excuse me that I write to you in English. Both German and English are not quit $[e]$ familiar to me and I cannot express my thoughts and feelings in both of them so well as I could do it in my own language. But now I am obliged to speak english (there are already 2 weeks as I live in London) and I feel myself just now more at home in the domain of the english language than in that of the German.

Three weeks ago I visited you (Sunday 8 January) but after that I have had so many impressions and have so much seen that these three weeks seem to me to be like three months. Paris and London I never have seen before and I was a little astonished by the larg[e]ness

${ }^{1}$ F. Mehring, Deutsche Geschichte vom Ausgange des Mittelalters, Ein Leitfaden für Lehrende und Lernende. Berlin I gro/I I, 
of these cities. London really seems to be not a town but a whole independent state.

But if even it would be not 3 or 4 weeks ago but 3 or 4 years ago that I have seen you - even in such case I should never forget your fr[i]endly home, your family and your kindness towards me. I am very sorry that I could not express to you in my own language how much I am pleased to make acquaintance with you. The expressions of a language which is not quite familiar always seem to be a little strange and not suitable.

The weather in London is not bea[u]tiful at all and especially at the beginning of my acquaintance with London, just when I came. There was once such a dark day like night.

I think that only in the cases of a pressing necessity one could live in London.

Berlin comparing with London seems to me to be very bright, sunny and clean.

I am very glad to begin to study in the British Museum which is the best library I have seen.

I greet you and your wife mit Sozialdemokratischem Gruss

Yours Sincerely

V. Adoratsky

III

München 14. XII. I917.

Sehr geehrter Herr Kautsky!

Entschuldigen Sie, bitte, dass ich Sie störe. Aber die jetzige ungewöhnliche Zeit und meine dadurch bestimmte Lage entschuldigen vielleicht einigermassen meine Aufdringlichkeit. Sie erinnern [sich] vielleicht, ich schrieb Ihnen vor etwa 6 Jahren und war sogar bei Ihnen. Die Rede war damals über theoretische Fragen, jetzt handelt es [sich] um eine praktische. Ich bin hier Zivilgefangener. Im Sommer I 914 kam ich mit meiner Frau und Tochter nach Deutschland zwecks Kur. Im Bad Reichenhall hat uns der Krieg überrascht.

Abzureisen gelang uns nicht, ich wurde Zivilgefangener, meine Frau und Tochter blieben bei mir. Wir wohnten zunächst in Bad Reichenhall, dann in Wörishofen und schliesslich seit October 1916 in München. Die Revolution erweckte die Hoffnung auf Rückkehr, aber die Hoffnung bleibt bis jetzt unerfüllt. Die jetzige[n] Verhand-

Im Original: und dadurch bestimmte meine Lage. 
lungen ${ }^{1}$ werden natürlich verschleppt, und ich bin in grosser Angst, [sie] werden kein Resultat bringen. Da meine politische[n] Freunde und Gesinnungsgenossen jetzt die Macht in den Händen haben, könnte ich vielleicht ausgetauscht werden. Aber wie muss ich handeln weiss ich nicht und fürchte, meine Bemühungen werden fruchtlos [bleiben]. Vielleicht können Sie mir einen Rat geben, ich habe hier fast gar keine Bekannten. Ich bitte nochmals um Entschuldigung, aber mein[e] Sehnsucht jetzt in Russland zu sein ist so gross, dass ich will alles versuchen. ${ }^{2}$

Mit besten Grüssen

Hochachtungsvoll W. Adoratsky Schellingstr. $58^{\mathrm{I}}$ München

1 Gemeint sind die Friedensverhandlungen in Brest-Litowsk.

${ }^{2}$ Kautskys Reaktion auf diese Bitte ist nicht bekannt. Adoratskij wurde auf Betreiben der im April 1918 in Berlin eingetroffenen sowjetrussischen Mission unter Leitung A. A. Ioffes im August 1918 die Heimreise gestattet. 